\title{
Process Model for Honing Larger Gears
}

\section{Fritz Klocke, Markus Brumm and Marco Kampka}

Hard finishing technology, e.g. - honing — is used to manufacture high-performance gears. Gear honing is primarily used to hard finish small- and medium-sized automotive gears. And yet trials have shown that gears with a module larger than $m_{n}=4 \mathrm{~mm}$ can also be honed efficiently, but problems often occur due to unstable process design. In this paper a model to improve the process design is described.

\section{Introduction}

Hard finishing technology is used to remove deviations from hardening, to machine tooth flank modifications and to meet quality requirements. The case hardening process is necessary to enable the gear to transmit high torque with smaller gears in high-power applications. In industrial applications, profile gear grinding, generating gear grinding and gear honing are most commonly used as hard finishing processes for gears. Each of these high-performance processes is using geometrically undefined cutting edges. Gear honing is primarily used to hard finish small- and medium-sized automotive gears with a module $m_{n}$ less than $4 \mathrm{~mm}$. One advantage is high economic efficiency as well as great surface quality. Trials have shown that gears with a module larger than $m_{n}=4 \mathrm{~mm}$ can also be honed efficiently. Problems occurred primarily in the reliability of the process - which leads to the conclusion that there is no problem, in principle, but rather a lack of knowledge of the honing process for large gears. Process parameters and tool specifications must be adjusted to derive the desired quality sought in honing larger gears.

To reduce time and effort, intensive trials with an empirical/physical process model were conducted. After discussing the theoretical process analysis, this paper focuses on practical process analysis based on force and vibration measurements.

\section{Challenge}

Initial attempts to scale up the process parameters from small to large gears have shown that this is not possible for all components. These results suggest that there is a lack of suitable process parameters and design data for honing largemodule gears. This is due to the kinemat-

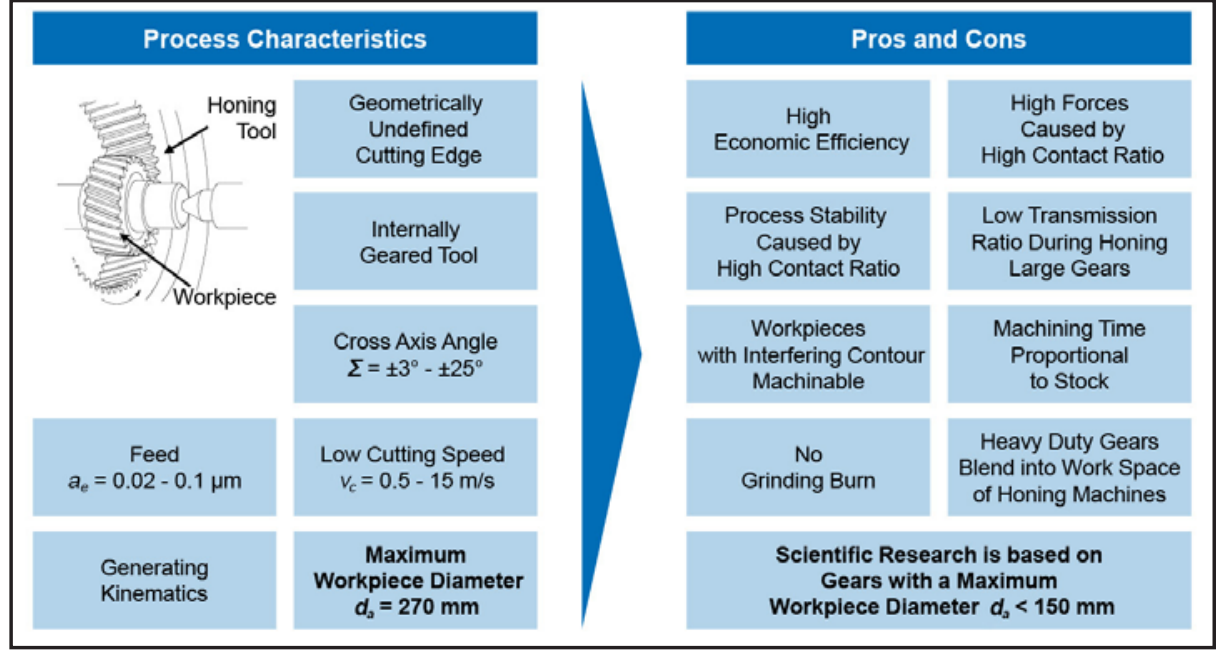

Figure 1 Process characteristics of gear honing.

ics of the gear honing process, where a large number of variables impart added complexity to the process. Additionally, small transmission ratios and high overlaps occur between the workpiece and honing tool for large gears (Fig. 1).

High overlaps lead to large spindle moments, which are necessary to cut a high amount of material as large contact zones occur. Secondly, the gear ratio between honing tool and workpiece becomes smaller due to the limited space, thus the machined volume for each tooth of the honing tool increases and the lifetime of the honing tool decreases, respectively, as the dressing cycles shorten.

Another process characteristic is that an internally toothed tool with geometrically undefined cutting edges meshes under a cross-axis angle with an externally toothed workpiece. Compared to other grinding processes, these cutting speeds are very low and barely reach up to $v_{c}=15 \mathrm{~m} / \mathrm{s}$ so that thermal structural damage can be avoided on the workpiece. Another advantage is that workpieces with interfering contours can be machined and that honing machines are already available in the industrial envi- ronment of large-module gears. A disadvantage of the honing process is the very low infeed - and thus the processing time - increases in proportion to the allowance.

In addition to process-related limits, the maximum size of the workpieces to be processed is limited to a tip diameter of $270 \mathrm{~mm}$ by the current honing machine generation, as the machines can hold honing with a maximum outside diameter of $400 \mathrm{~mm}$. Given that honing is mainly found in the automotive industry, most of its scientific study is based on gears with a tip diameter of less than $150 \mathrm{~mm}$.

\section{Research Objective and Approach}

Due to the existing challenges, a research project to optimize the honing process for large gears was initiated (Fig. 2).

The objective of this research project is to increase efficiency and process safety during honing of large-module gears. The objective of this report is to build an empirically physical process model. The model is intended to increase the efficiency and process reliability for honing and to contribute to an improved 
understanding of the process. The procedure for this is explained throughout the report. First of all, specific values for different honing process designs are generated on the basis of evaluated process designs chosen for practical experiments to allow direct linking of the results. To increase the amount of information gathered in the practical investigations, the test machine was equipped with additional measurement devices that make it possible to detect both the process resulting forces and the vibrations of various machine components.

For this purpose, the test workpiece and the honing tool designs are presented for different cross-axis angles in (Workpiece and Honing Tool Designs) before the results of the analytical calculation for the presented workpiece honing ring combinations are explained. The test machine and the measuring chain are then shown before the results of the force measurements are discussed in (Empirical Process Analysis section).

\section{Workpiece and Honing Tool Designs}

The workpiece used for the investigations presented in this paper is a transport transmission gear. The number of teeth is $z=47$, with a module of $m_{n}=4.55 \mathrm{~mm}$ and a normal pressure angle of $\alpha_{n}=21^{\circ}$. The helix angle is $\beta=18^{\circ}$. The gear has a width of $b=46.92 \mathrm{~mm}$ and a tip diameter of $d_{a}=231.75 \mathrm{~mm}$.

For the analytical calculations and honing trials, four different honing tool designs are used (Fig. 3); four different cross-axis angles were chosen. The complex relationships during honing require that additional geometric variables must be changed by adjusting the cross-axis angle $\Sigma$. This is due to the relationship that the sum of the helix angle of the tool and workpiece forms the cross-axis angle
Manufacturing Large Gears

Honing large gears becomes difficult due to:

- Low transmission

- High cover ratio

- Target values have negative interactions among each other

\section{Project for Optimization of the Honing Process of large Gears} sponsored by Eurostars / BMBF

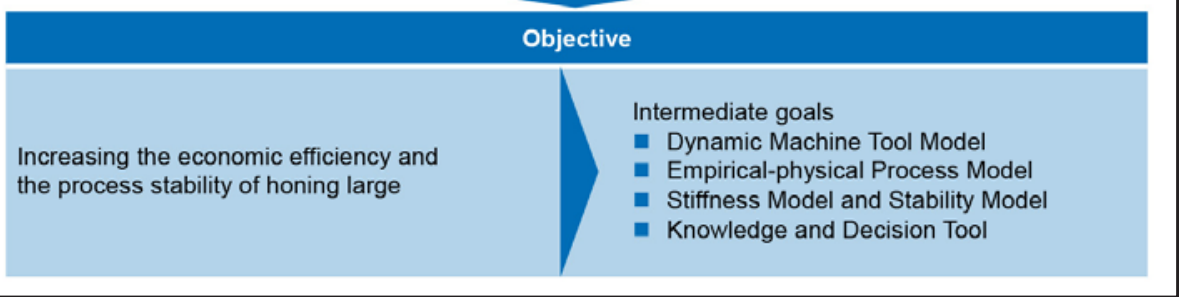

Figure 2 Research objective.

\begin{tabular}{|c|c|c|c|c|}
\hline & HR1 & HR2 & HR3 & HR4 \\
\hline$\Sigma\left[{ }^{\circ}\right]$ & -15 & -8 & -3 & +8 \\
\hline$\beta_{H R}\left[{ }^{\circ}\right]$ & 2.599 & 9.818 & 14.961 & 26.24 \\
\hline $\mathrm{z}_{H R}[-]$ & 75 & 75 & 73 & 67 \\
\hline $\mathbf{d}_{\mathrm{a}, H R}[\mathrm{~mm}]$ & 330.994 & 335.791 & 332.498 & 327.35 \\
\hline $\mathbf{d}_{f, H R}[\mathrm{~mm}]$ & 354 & 358.4 & 355.5 & 350.4 \\
\hline a [mm] & -60.779 & -62.884 & -61.2937 & $-58,380$ \\
\hline $\begin{array}{l}\mathrm{HR}=\text { Honir } \\
\text { WST }=\text { Work }\end{array}$ & & & & \\
\hline
\end{tabular}

Figure 3 Honing tool designs.

$\Sigma$. At a constant helix angle $\beta$ of the workpiece, a change in the cross-axis angle $\Sigma$ is followed by a change of the helix angle of the honing tool $\beta$. Therefore, the number of teeth $z$ needs to be adjusted - resulting in a tip and root diameter change. This in turn must be compensated for by an adjustment of the center distance $a$. The corresponding honing tool designs can be found in Figure 3. In addition to the reference tool for this workpiece with a cross-axis angle of $\Sigma=-8^{\circ}$, three other cross-axes were selected with pairwise different signs of the cross-axis angle at $\Sigma=-8^{\circ}$ and $\Sigma=+8^{\circ}$. This leads to the same cutting speed at the same rotational speed for this pairing.

\section{Analytical Modelling of the Gear Honing Process}

The cutting speed $v_{c}$ is mainly responsible for machining the stock. The cutting speed is made up of the three components - lateral gliding speed $v_{g H}$, longitudinal sliding speed $v_{g L}$ and oscillation speed $v_{\text {osc }}$. The oscillation speed is by a factor of $10^{-3}$ less than the other two components, and can therefore be ignored, so that the cutting speed may be described by the scalar addition of the lateral gliding speed $v_{g H}$ and the longitudinal sliding speed $v_{g L}$. The lateral gliding speed $v_{g H} \mathrm{can}$ be calculated out of the radii of curvature and the angular velocities of the meshing parts, while the longitudinal sliding speed $v_{g L}$ depends on the circumferential speed, helix angle and cross-axis angle. From cutting speed $v_{c}$ and feed speed $v_{\text {rad }}$, the cutting performance is derived. The cutting performance can be used as a measure for process efficiency, which is the relevant value for honing process optimization.

For noise optimization, the cutting angle is the decisive factor, according to Köllner. An optimum noise behavior occurs when the machining marks are orthogonal to the lines of contact between wheel and pinion, with the cutting angle matching the angle of the machining marks (Ref.1). Schweikert, however, determines that the cutting angle has no influence on the noise characteristics of gears and that it is sufficient that either pinion or wheel is honed (Ref.2). The path of the line of contact depends mainly on the helix angle of the gear. The contact line length and orienta- 
tion are two other evaluation criteria. The contact line length can be used as a measure of the contact area between tool and workpiece. Due to the constant contact force, a larger contact area leads to lower surface pressure. Thus, cutting material is more difficult because the necessary force for chip formation remains constant on the workpiece, but the processing power is distributed over a larger area. Consequently, cutting performance decreases with an increase of contact line length.

To a particular extent, this problem affects honing of large gears because an increase in size is usually associated with an increase of gear width. This is followed in turn by longer contact lines and an increase of material volume, which leads to a reduction of cutting performance - which is critical for the economic efficiency of the process.

Due to the independence of the contact line orientation from cutting angle and machining marks, the orientation of the contact lines has no direct influence on the process result, but does so indirectly via the process dynamics on the surface finish. Nevertheless, the orientation can be used as an indicator for process behavior. If machining marks occur, which are oriented parallel to the axis, the whole contact line length comes into contact very quickly. The expected effect is similar to a premature tooth contact and can lead to unsatisfactory process results. If the contact lines are more steeply oriented, the contact comes gradually and thus exerts no negative effect on the process (Ref. 1). The disadvantage of these kinematics is that the contact line length changes continuously during honing. This leads to different machining forces and torques that can negatively affect the process itself. Therefore, an optimization of the contact line orientation must be made in order to achieve an optimum result.

In addition to the previously conducted analysis of the contact line length for a tooth flank, the contact line length must be examined at the same time on both flanks of each engaged tooth. To describe the change in contact line length, a suitable value was found in the difference of total contact line length between both flank sides. First, the summation of contact length of all engaged teeth is

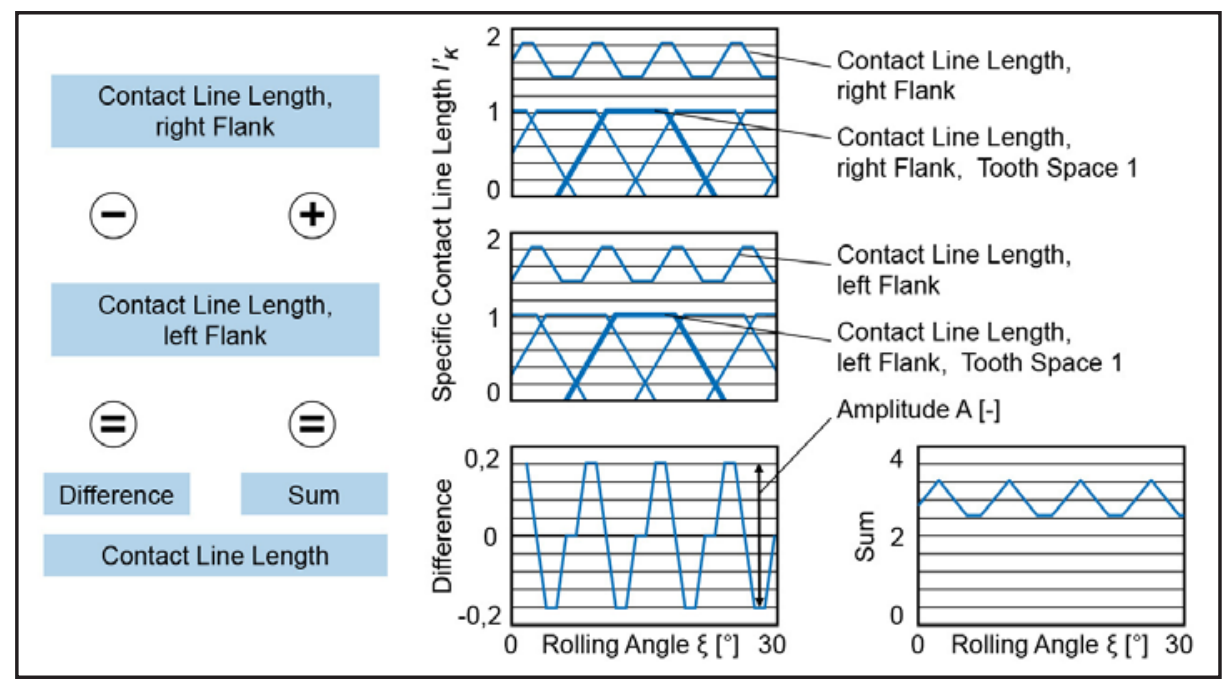

Figure 4 Difference and sum of the length of line of contact.

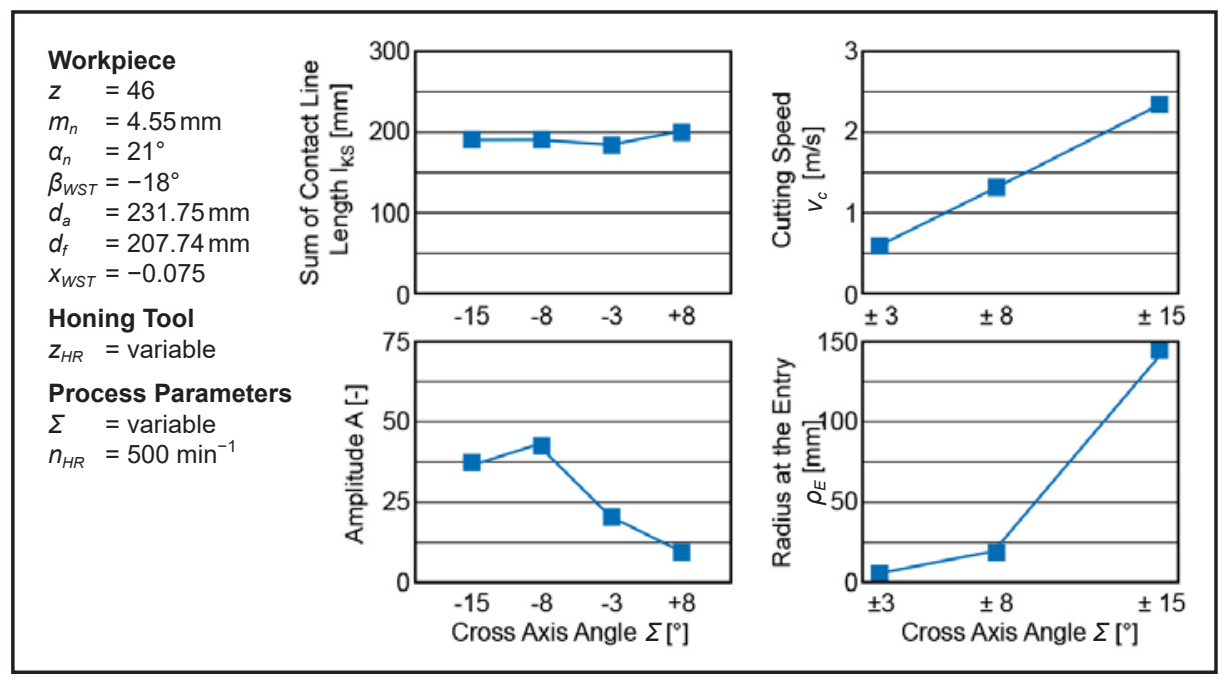

Figure 5 Influence of the cross-axis angle on the characteristic values.

separately formed for the left and right flanks to get the total contact line length. Subsequently, the total contact line length of the left and the right flank (Fig.4) are subtracted from each other. At the considered helical gear, there is an angle on the contact lines, and thus, as already explained, a change in contact line length over the tooth profile. This continuous change in the contact conditions between left and right flank results in a change of torque. An exact machining of the desired topology is therefore not possible.

To describe this effect, the difference in total contact line length will be used. The total contact line lengths of the left and right flank can be regarded as phaseshifted oscillations - with the same frequency and amplitude. The difference in contact line length is shown in Figure 4 ; each difference of the total contact line length from the zero point changes torque during honing (Ref. 1).
In the following, the influence of the cross-axis angle on the process at a constant rotational speed of $n=500 \mathrm{~min}^{-1}$ and a constant feed of $f=0.06 \mu \mathrm{m}$ is investigated. The simulated total contact line length $l_{K S}$ as the sum of contact line lengths of left and right flank of the workpiece is influenced by the cross-axis angle (Fig. 5).

Within the group of negative crossaxis angles no systematic change in the total contact line length was identified. The value at $\Sigma=-15^{\circ}$ and $\Sigma=-8^{\circ}$ is $l_{K S}=188 \mathrm{~mm}$, and decreases to $l_{K S}=182 \mathrm{~mm}$ for $\Sigma=-3^{\circ}$. In contrast to this group the values for the total contact line length are increasing for positive cross-axis angles. The total contact line length increases to $l_{K S}=199 \mathrm{~mm}$ at $\Sigma=+8^{\circ}$. Due to the higher total contact line length for cross-axis angle with a positive sign, it is assumed that the cutting forces will be increased for these 
process parameters.

A positive effect of using positive cross-axis angle is indicated by the amplitude $A$. The amplitude $A$ is defined in this report as the unit less value of the difference between minimum and maximum of total contact line difference between left and right flank. From the amplitude $A=25$ at $\Sigma=-15^{\circ}$, the amplitude increases to the significant maximum $A=42$ at $\Sigma=-8^{\circ}$ before decreasing to $A=22$ at $\Sigma=-3^{\circ}$. In the area of positive cross-axis angle, the level is considerably lower, and the values of the amplitude reach a minimum of $A=10$ at $\Sigma=+8^{\circ}$. Low amplitude ensures a stable process without much disturbance in torque (Ref.1). The results shown therefore point out that a positive cross-axis angle can be a possible solution if there are any vibration problems machining this component. At large cross-axis angles, the radius at the entry of the abrasive grain into the workpiece gets bigger and can result in a shallow immersing of the abrasive grains into the workpiece. This ensures that a high theoretical chip length $l_{s}$ is generated. The chip length will be significantly reduced because the minimum cutting thickness is achieved rather late by the flat path, and a lot of energy will be flowing into plastic deformation of the workpiece material. Therefore the cutting process at a cross-axis angle of $\Sigma= \pm 15^{\circ}$ is energetically classified as unfavorable. However, the flat grain path can have a positive effect on the wear behavior of the honing tool, since the collision energies are small and lead to longer tool life (Ref.3). Large, effective direction angles are energetically advantageous because they allow good stock removal. This is achieved because the abrasive grains will penetrate the workpiece material more steeply via a decrease in radius of curvature. The stresses on the grain as it enters the workpiece will increase so that a decrease of honing tool lifetime is possible (Ref.4).

\section{Experimental Set-Up}

The machine used for the trials in this paper is a modified gear honing machine (HMX-400) from Fässler. In addition to the standard integrated force measurement in $x$-direction to hone gears by force-controlled feed, a variety of other sensors was integrated into the machine

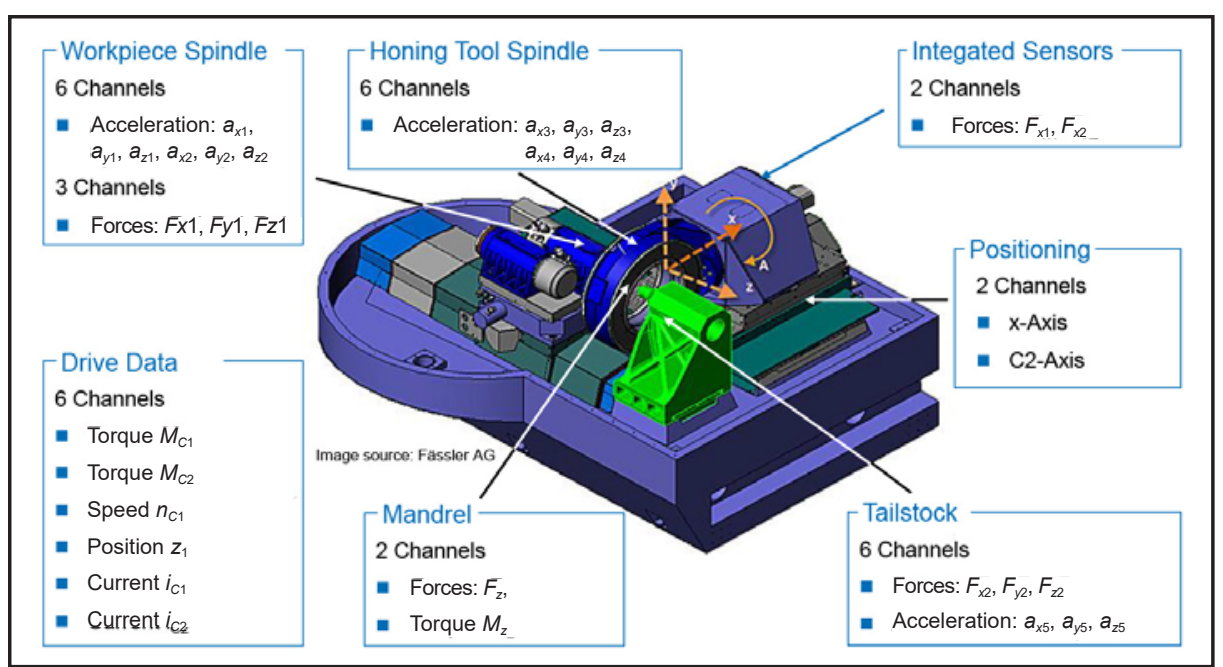

Figure 6 Trial set-up.

(Fig. 6).

The workpiece side force is measured via pre-loaded sensors from Kistler. These were placed in the flux-of-force of the workpiece spindle and in the flux-offorce of the centering point. Accordingly, this arrangement allows receiving the forces in the three directions of a stationary coordinate system, corresponding to the coordinate system of the machine. Another sensor for measuring the force in $z$-direction is located in the mandrel and transfers the data without contact via a telemetry unit. The internal drive data is used to detect the torque of the spindle, the rotational speed of the tool spindles and the complementary current. In addition, the positions of $z 1$ and $x$-axis are recorded in parallel. The measurements with the calibrated force sensors are presented in (Empirical Process Analysis section).

Since gear honing is a highly dynamic process, various influences can lead to vibrational excitation of the machine. To measure those vibrations, three-axis acceleration sensors were built into the spindles and centering point. In each case, two sensors were used in the spindle and the centering point, which, by their arrangement, allows us to draw conclusions about any torques. Based on this data it should be possible to describe the process extensively and to couple process forces and vibrations with a machine model. By integrating such a massive measurement system, especially in the power flow in case of the force sensors, the rigidity of the machine is reduced. Therefore, the results need to be particularly scrutinized in the range of the exci- tation behavior of the machine and cannot be transferred directly to the serial machine.

The sensor output voltages are converted by charge amplifiers to a range from $U= \pm 10 \mathrm{~V}$, which can be received by the measuring card. As a measuring card serves a modular system from National Instruments that allows synchronized recording of 32 channels with a resolution of 16 bit, the scanning of the sensors is carried out with a frequency of $15 \mathrm{kHz}$ and thus with a significantly higher frequency than the tooth meshing frequency of the presented process. Furthermore, to avoid aliasing and to reduce the data rate to a level that's easy to handle, the measurement datasets are hardware-based, pre-filtered, clocked down to a frequency of about $5 \mathrm{kHz}$ and transmitted in real time to an external disk.

The trace of a clocked-down $5 \mathrm{kHz}$ signal is plotted over time (Fig. 7, top). The yet very high frequency continues complicating the interpretation of the force signals, so the signal must be further processed. For this purpose, the signals are reduced in Matlab to a frequency of $25 \mathrm{~Hz}$ and subsequently formed by the moving average of 100 readings. The resulting trace is shown (Fig. 7, bottom). In addition to the consideration of the mean values, the dynamics of the measured amplitudes and frequencies must be addressed.

To reduce the amount of data, the datasets were divided manually due to a lack of an automated cutting recognition so that only main machining time is considered and the non-contact time and spark out are not incorporated within 
the results. These areas were determined individually for each experimental point, as the process execution with a constant feed rate lead to varying processing times because of the fluctuating distortion due to the heat treatment and soft machining of the components.

\section{Empirical Process Analysis}

To simplify the interpretation of the results - and to eliminate as many variables as possible - only the measurement results of two tool designs with a cross-axis angle of $\Sigma=-8^{\circ}$ and $\Sigma=+8^{\circ}$ will be compared in the following. The analytical evaluation of these designs showed a significant difference between the amplitude parameters and therefore should be favorable to analyze the influence of the amplitude parameter on the process. The process parameters used in the trials were derived from a constant cutting speed of $v_{c}=3 \mathrm{~m} / \mathrm{s}$ at the pitch diameter of the workpiece. The resulting rotational speeds of the honing tool were $n=1,783 \mathrm{~min}^{-1}$ for a crossaxis angle of $\Sigma=+8^{\circ}$ and $n=1,872^{-1}$ for a cross-axis angle of $\Sigma=-8^{\circ}$. The feed rate was $f=0.09 \mu \mathrm{m}$ for both processes. As an example, the resulting radial forces in $x$ direction are shown (Fig. 8). The force readings follow a characteristic curve. First, a progressive increase in force is applied by establishing contact and slowly machining first feed marks and generating cut deviations from the preprocessing. This is followed by an approximately linear increase in force. This shows that during machining, no stable process is reached, where a constant force would be assumed. The force curves for both crossaxis angles are similar.

The maximum force for a cross-axis angle of $\Sigma=-8^{\circ}$ is $-1,800 \mathrm{~N}$ and therefor a little bit higher as for a cross-axis angle of $\Sigma=+8^{\circ}$ with a maximum radial force of $-1,650 \mathrm{~N}$. But the approximately five percent lower rotational speed at a cross-axis angle of $\Sigma=+8^{\circ}$ is followed by less machined material. Considering the linear increase in force, the maximum force would be higher for the same material removed. The difference between the maximum forces would decrease to an insignificant amount and can therefore be ignored.

An influence of the specific value of the total contact length, which was at

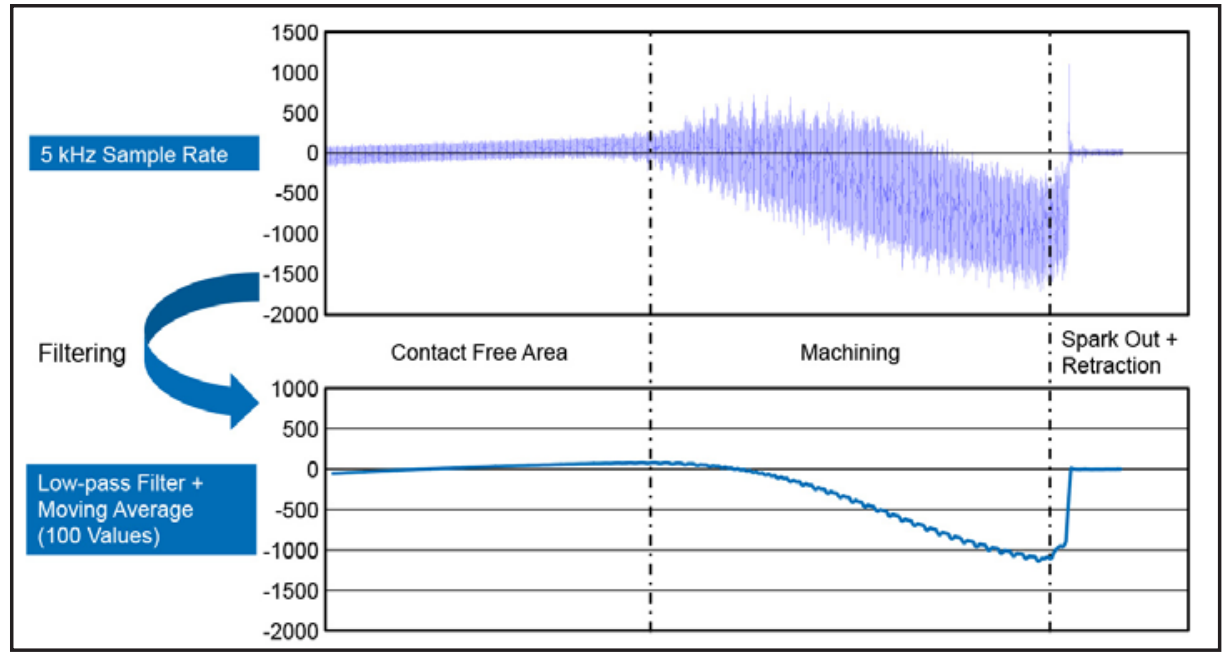

Figure 7 Signal processing.

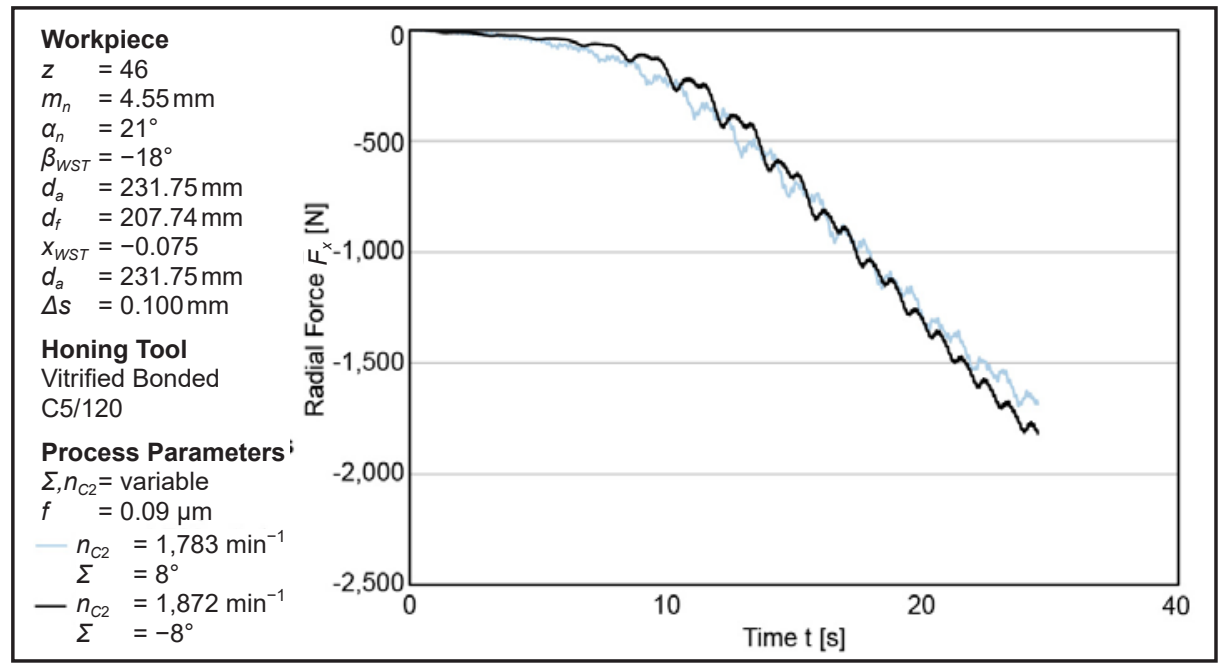

Figure 8 Influence of the cross-axis angle on the radial force one-half.

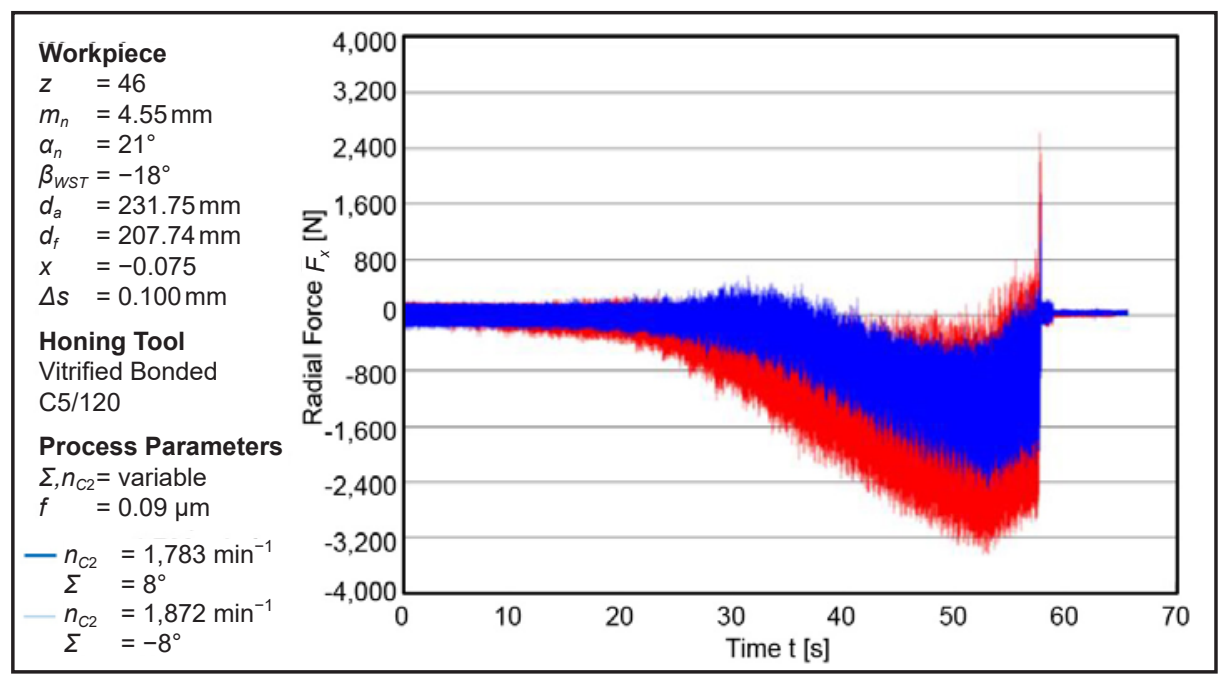

Figure 9 Influence of the cross-axis angle on the radial force $2 / 2$. 
$l_{K S}=188 \mathrm{~mm}$ for $\Sigma=-8^{\circ}$ and $l_{K S}=199 \mathrm{~mm}$ for $\Sigma=+8^{\circ}$, on the maximum force was not found. The difference between the analyzed tool designs in case of total contact length seems too small to be found in the measurements. But comparing the quality of the honed gears machined by using the whole variety of tools mentioned before to the average or maximum forces shows no correlation. Therefore, the vibration during gear honing will be in focus in the following. Figure 9 shows the force reading during honing with less filtering than before.

Comparing the force amplitudes near the end of the process, a force amplitude of 2,000 $\mathrm{N}$ can be found for a crossaxis angle of $\Sigma=+8^{\circ}$ and a force amplitude of 3,200 $\mathrm{N}$ for a cross-axis angle of $\Sigma=-8^{\circ}$. Therefore, the influence of the contact line length difference shown in (Analytical Modelling of the Gear Honing Process) is confirmed. The amplitude value of $A=42$ at $\Sigma=-8^{\circ}$ is significantly higher than the amplitude of $A=10$ at $\Sigma=+8^{\circ}$. Since there is a factor of more than four between the amplitude values and only a factor of 1.5 between the force readings, it is shown that there is no linear correlation and only a qualitative prediction is possible. The difference in force amplitude leads to a bad workpiece geometry for a cross-axis angle of $\Sigma=-8^{\circ}$ whereas a good workpiece geometry for a cross-axis angle of $\Sigma=+8^{\circ}$ was achieved. Changing the cross-axis angle to $\Sigma=+8^{\circ}$ made it possible to machine the part 50\% faster with a good workpiece geometry compared to the reference process design with a cross-axis angle of $\Sigma=-8^{\circ}$.

\section{Summary and Outlook}

Gear honing is an established hard finishing process for small gears. Although modern gear honing machines are capable of honing gears with a tip diameter up to $d_{a}=270 \mathrm{~mm}$, gear honing could not become accepted in the industry for hard finishing gears with a tip diameter between $d a=150$ and $d_{a}=270 \mathrm{~mm}$. There are positive examples of parts that size that are honed successfully, but the process often missed the needed stability to generate the demanded quality reliably. To date, the scientific investigations of honing large gears are insufficient.

It was the research objective of this paper to develop an approach for an empirical-physical process model. The analyses of the process forces in connection with the process parameters and the analytical calculated characteristic values were shown. In an ideal process, force and torque would seek a stationary state - which was not reached in the trials. The cross-axis angle variation during the trials has shown - in comparison with the characteristic values of the analytical calculation - that the characteristic values are partially meaningful. On the one hand, for the total contact line length, no correlation was found regarding the maximum forces during honing. But it was also shown that there is no direct correlation between the maximum force and gear quality. Therefore, this specific value is not useful. On the other hand, the amplitude parameter $A$, as the difference between the contact line length between the right and the left flank, can be used to give a quantitative predication of the force amplitudes in the process that can be directly related to gear quality. A huge increase of productivity is possible by changing the cross-axis angle and therefore reducing the force amplitudes during honing.

This analysis has shown great potential for the presented workpiece. Additional gear designs must be analyzed and compared to the prediction values to validate the approach.

\section{References}

1. Köllner, T. "Verzahnungshonen -

Verfahrenscharakteristik und Prozessanalyse," Dissertation, 2000.

2. Schweikert, S. "Beitrag zur Entwicklung des Leistungshonens von Verzahnungen," Dissertation, 2001.

3. Klocke, F., C. Gorgels and V. Vasiliou. Abricht- und "Bearbeitungstechnologie beim Verzahnungshonen mit Keramischgebundenen Werkzeugen," 2009.

4. Klocke, F. and W. König. "Fertigungsverfahren, Band 2: Schleifen, Honen, Läppen,” 2005.

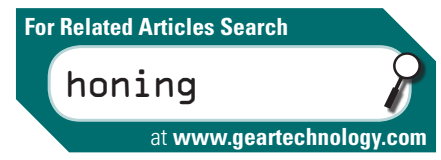

Markus Brumm, a RWTH graduate with a degree in mechanical engineering, began his career in 2005 as a research assistant in gear investigation at the Laboratory for Machine Tools and Production

Engineering (WZL) of the RWTH Aachen. He subsequently became that group's team leader in 2010. In November 2014, he joined Klingelnberg $\mathrm{GmbH}$ as manager of the company's Technology Center.

Prof. Dr.-Ing. Dr.-Ing. E.h. h.c. Dr. h.c. Fritz Klocke studied manufacturing engineering at the TU Berlin, was a research fellow there at the Institute for Machine Tools and manufacturing Technology until 1981, and then as head engineer until 1984, receiving his engineering doctorate in 1982. Klocke worked in industry from 1984 until 1994 at Ernst Winter \& Sohn in Hamburg. On January 1 , 1995 he was called to the RWTH Aachen as Professor of Manufacturing Engineering Technology and has since then been Chair of Manufacturing Technology, co-director of the WZL Laboratory for Machine Tools \& Production Engineering at the RWTH Aachen and head of the Fraunhofer Institute for Production Technology IPT in Aachen. Klocke was awarded the Otto-Kienzle Memorial Coin in 1985 by the Manufacturing Engineering University Group. The title, “Dr.Ing. E.H," was bestowed upon Klocke by the University of Hannover in 2006 for his outstanding achievements in science, his efforts in the industrial implementation of a broad range of manufacturing techniques, and for his commitment to numerous scientific committees. The title "Dr. h. c." was awarded him in 2009 by the University of Thessaloniki and in 2010 by Keio University in Tokyo for his achievements in production science, his engagement in international cooperation, and his benefits as a teacher and supervising tutor of student engineers.

Dipl.-Ing. Marco Kampka is a scientific research assistant at the Laboratory for Machine Tools and Production Engineering (WZL) of RWTH Aachen University, Chair of Manufacturing Technology. After one year of military service (2004), Kampka earned from 2005-

2012 his degrees in mechanical engineering at Aachen, with a focus on production engineering, in particular the hard finishing of gears, gear honing and surface properties. 